\title{
Rethinking the Crit
}

PATRICK FLYNN - MIRIAM DUNN

- MARK PRICE - MAUREEN O'CONNOR

TU Dublin UCD SAUL CIT 
Assessment in architecture and creative arts schools has traditionally adopted a 'one size fits all' approach by using the 'crit', where students pin up their work, make a presentation and receive verbal feedback in front of peers and academic staff. In addition to increasing stress and inhibiting learning, which may impact more depending on gender and ethnicity, the adversarial structure of the 'crit' reinforces power imbalances and thereby ultimately contributes to the reproduction of dominant cultural paradigms.

Our collaboration on an alternative to the traditional model was supported by the Teaching \& Learning National Seminar Series fund which helped us organise an international symposium to debate the 'crit' in 2016. We have recently been awarded further funding which has allowed us to pilot alternative feedback methods.

Our proposal will add blended learning to new assessment methods which we piloted in 2017/8, in a radical approach challenging the dominant pedagogical theory and practice in architecture internationally and should transform feedback and assessment methods. Feedback on the pilot from students, academic staff and external examiners has been extremely positive.

We will expand this pilot across four HEls, review emergent best practice abroad and bring international experts to evaluate and develop the approach. While our focus will be on architecture, it will be relevant to other creative disciplines which use the 'crit' method. We will explore digital approaches to support student reflection. We will explore how this method can work across all creative art education.

This approach has the potential to give students greater agency, enhanced critical faculties, professional skills and resilience, supporting transitions into and out of third level.

Our first pilot has shown that this new feedback and assessment method uses staff time in a more efficient and effective manner, with the student becoming central to the learning process. Our current research has been peer reviewed by leading academics in the field and at this conference we will be able to present our findings for the first time. 